\title{
The size of metastatic foci and lymph nodes yielding false- negative and false-positive lymph node staging with positron emission tomography in patients with lung cancer
}

Hiroaki Nomori, MD, $\mathrm{PhD}^{\mathrm{a}}$

Kenichi Watanabe, $\mathrm{MD}^{\mathrm{a}}$

Takashi Ohtsuka, MDa

Tsuguo Naruke, MD, $\mathrm{PhD}^{\mathrm{a}}$

Keiichi Suemasu, MD, $\mathrm{PhD}^{\mathrm{a}}$

Kimiichi Uno, MD, PhD ${ }^{\mathrm{b}}$

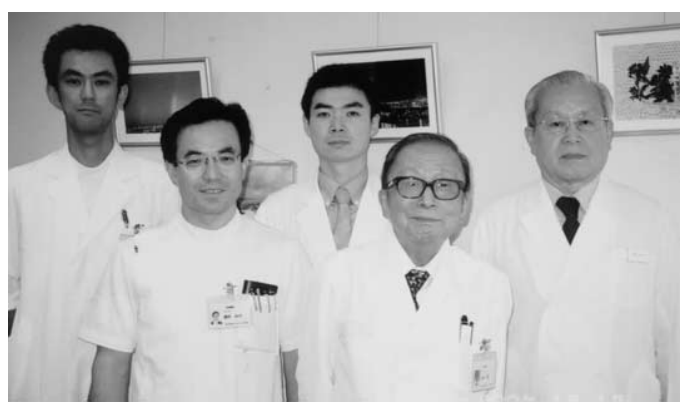

Left to right: Watanabe, Nomori, Ohtsuka, Suemasa, Naruke
Background: We examined the sizes of lymph nodes and metastatic foci within the lymph nodes that affect false-positive and false-negative lymph node staging by positron emission tomography in lung cancer.

Methods: Preoperative positron emission tomography and computed tomography scans were performed for 564 lymph node stations in 80 patients with peripheral-type lung cancer. The sizes of both the lymph nodes and the metastatic foci within the lymph nodes were measured, and these measurements were compared with those obtained with positron emission tomography scanning. To establish general sizes of metastatic foci within the lymph nodes, 277 metastatic lymph nodes in operative specimens previously resected from another 111 patients with lung cancer were examined as a control.
From the Department of Thoracic Surgery, Saiseikai Central Hospital, Tokyo, Japan, ${ }^{\text {a }}$ and Nishidai Clinic, Tokyo, Japan. ${ }^{\mathrm{b}}$

Received for publication June 13, 2003; revisions requested July 14,2003 ; revisions received July 23, 2003; accepted for publication Aug 11, 2003.

Address for reprints: Hiroaki Nomori, MD, Department of Thoracic Surgery, Saiseikai Central Hospital, 1-4-17 Mita, Minato-ku, Tokyo 108-0073, Japan (E-mail: hnomori@qk9.so-net.ne.jp).

J Thorac Cardiovasc Surg 2004;127:1087-92 $0022-5223 / \$ 30.00$

Copyright $\odot 2004$ by The American Association for Thoracic Surgery

doi:10.1016/j.jtcvs.2003.08.010
Results: The sensitivity was significantly higher for positron emission tomography than for computed tomographic scanning $(P=.026)$. The sizes of metastatic foci within lymph nodes that showed false-negative $(\mathrm{n}=8)$ and true-positive $(\mathrm{n}=28)$ with positron emission tomography ranged from 0.5 to $9 \mathrm{~mm}(3 \pm 1 \mathrm{~mm})$ and from 4 to $18 \mathrm{~mm}(10$ $\pm 3 \mathrm{~mm}$ ), respectively $(P<.001)$. None of the metastatic foci smaller than $4 \mathrm{~mm}$ could be detected with positron emission tomography scanning. The review of the 277 previously resected metastatic lymph nodes showed that 89 (32\%) had metastatic foci smaller than $4 \mathrm{~mm}$. The sizes of true-positive $(\mathrm{n}=28)$ and false-positive $(\mathrm{n}=10)$ lymph nodes ranged from 6 to $15 \mathrm{~mm}(10 \pm 2 \mathrm{~mm})$ and from 9 to $16 \mathrm{~mm}(12 \pm 2 \mathrm{~mm})$, respectively $(P<.01)$. None of the false-positive lymph nodes was smaller than $9 \mathrm{~mm}$.

Conclusions: Although positron emission tomography was superior to computed tomography scanning in lymph node staging in lung cancer, positron emission tomography was unable to distinguish metastatic foci smaller than $4 \mathrm{~mm}$, which were not unusual sizes for lymph node metastases in lung cancer. Positive lymph nodes with positron emission tomography smaller than $9 \mathrm{~mm}$ are likely to be true-positive rather than false-positive.

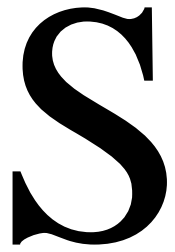

everal diagnostic procedures are used for preoperative staging of patients with lung cancer, including computed tomography (CT), magnetic resonance imaging, positron emission tomography (PET), mediastinoscopy, and thoracoscopy. Of the noninvasive procedures, $\mathrm{CT}$ remains the standard procedure for $\mathrm{N}$ staging in lung cancer. However, CT scanning is not sufficiently sensitive or specific for diagnosing lymph node metastasis ${ }^{1}$; because size is the only criterion used to differentiate benign from malignant lymph nodes, lymph nodes $1 \mathrm{~cm}$ or larger are considered abnormal. 
TABLE 1. Characteristics of patients with peripheral lung cancer who underwent positron emission tomography and computed tomography

\begin{tabular}{lc}
\hline Variable & Data \\
\hline Age (y) & $65 \pm 12$ \\
Sex & \\
Male & 57 \\
Female & 23 \\
Tumor size (cm) & $3.3 \pm 2.0$ \\
Tumor location & \\
Right upper lobe & 27 \\
Right middle lobe & 5 \\
Right lower lobe & 10 \\
Left upper lobe & 21 \\
Left lower lobe & 17 \\
Histology & \\
Adenocarcinoma & 57 \\
Squamous cell carcinoma & 14 \\
Large cell carcinoma & 3 \\
Small cell carcinoma & 3 \\
Carcinosarcoma & 2 \\
Adenosquamous carcinoma & 1 \\
Pathologic N stage & \\
N0 & 56 \\
N1 & 10 \\
N2 & 11 \\
N3 & 30 \\
Total & \\
\hline
\end{tabular}

In recent years, fluorodeoxyglucose (FDG)-PET scanning has been used for the staging of lung cancer. ${ }^{2-7} \mathrm{Be}-$ cause of the biological nature of FDG, FDG-PET has been reported to detect metastatic lymph nodes smaller than 1 $\mathrm{cm}$. A meta-analysis by Dwamena and colleagues ${ }^{1}$ of PET scanning of 514 patients in 14 studies showed that the mean sensitivity and specificity for PET scanning in $\mathrm{N}$ staging were 0.79 (range, 0.62 to 0.97 ) and 0.91 (range, 0.79 to 0.99 ), respectively, both of which were superior to those for CT scanning, ie, 0.60 (range, 0.25 to 0.89 ) and 0.77 (range, 0.44 to 0.95 ), respectively. The detection of small metastatic lymph nodes is, however, limited by the spatial resolution of the PET scanner. In this study, to determine the lower size limit of the metastatic lymph nodes that can be detected by PET scanning, we measured the sizes of metastatic foci in lymph nodes with true-positive (TP) and false-negative (FN) results, and these results were compared with the sizes of metastatic lymph nodes from another large series of lung cancer patients to determine the general frequency of sizes of metastatic lymph nodes. We also measured the sizes of TP and false-positive (FP) lymph nodes to determine the lymph node sizes that gave FP results on PET scan.

\section{Materials and Methods}

\section{Subjects}

Between December 2001 and May 2003, 85 patients with lung cancer prospectively underwent FDG-PET and CT scanning be- fore pulmonary resection with mediastinal lymph node dissection. Lung cancer was peripheral type in 80 patients and central type in 5. For this study, we excluded the 5 patients with central-type lung cancer, giving a total of 564 lymph node stations in the 80 patients with peripheral-type lung cancer who were evaluated. The histologic type of lung cancer was adenocarcinoma in 57 patients, squamous cell carcinoma in 14 , large cell carcinoma in 3 , small cell carcinoma in 3, carcinosarcoma in 2 , and adenosquamous carcinoma in 1 (Table 1). The pathologic N stages were N0 in 56, $\mathrm{N} 1$ in $10, \mathrm{~N} 2$ in 11 , and N3 in 3 . The lymph nodes were classified according to the original lymph node map of lung cancer. ${ }^{8}$ All patients underwent thoracotomy without mediastinoscopy, except for 3 patients with N3 disease. The 3 patients with N3 disease had primary tumors in the left lung, which were resected with contralateral lymph node dissection by median sternotomy.

\section{FDG-PET Scanning}

Patients were instructed to fast for at least 4 hours before intravenous administration of FDG. The administered dosage of FDG was $125 \mu \mathrm{Ci} / \mathrm{kg}(4.6 \mathrm{MBq} / \mathrm{kg})$ of body weight for nondiabetic patients and $150 \mu \mathrm{Ci} / \mathrm{kg}(5.6 \mathrm{MBq} / \mathrm{kg})$ of body weight for diabetic patients. PET imaging was performed approximately 60 minutes after intravenous administration of the FDG by using a POSICAM.HZL m-POWER (Positron Co, Houston, Tex). No-attenuation-corrected emission scans were initially obtained in 2-dimensional, highsensitivity mode for 4 minutes per bed position and taken from the vertical skull through to the mid thighs. Immediately thereafter, a 2-bed-position attenuation-corrected examination was performed, with 6 minutes for the emission sequence and 6 minutes for the transmission sequence at each bed position. The images were usually reconstructed in a $256 \times 256$ matrix by using ordered subset expectation maximization corresponding to a pixel size of 4 $\times 4 \mathrm{~mm}$, with section spacing of $2.66 \mathrm{~mm}$.

\section{N Staging by PET Scanning}

PET data were evaluated semiquantitatively on the basis of the contrast ratio (CR), which was obtained as follows. The regions of interest (ROIs) were placed in the lymph nodes and cerebellum. The highest activities in both the lymph node ROI (L) and the cerebellum ROI (C) were measured. The CR was calculated by $\mathrm{L} / \mathrm{C}$ in each lymph node as an index of FDG uptake. Receiver operating characteristic curves ${ }^{9}$ were constructed according to the $\mathrm{CR}$ value and pathologic diagnosis, and the cutoff value was determined for diagnosis of metastasis.

\section{N Staging by CT Scanning}

Spiral CT was performed by using the ProSeed SA (General Electric Medical Systems, Milwaukee, Wis). The following acquisition parameters were used: high voltage $(120 \mathrm{kV})$, tube load of $160 \mathrm{~mA}$, window level of -500 Hounsfield units (HU), and window width of $1500 \mathrm{HU}$. The entire thorax was scanned with 1 -cm-thick sections at 1 breath hold with maximum inspiration. The criterion of CT definition for suspected metastasis of the lymph node was a short-axis diameter of $1.0 \mathrm{~cm}$ or larger. 
TABLE 2. Characteristics of control of lung cancers with lymph node metastasis

\begin{tabular}{lr}
\hline Variable & No. \\
\hline Histology & \\
Adenocarcinoma & 72 \\
Squamous cell carcinoma & 26 \\
Large cell carcinoma & 10 \\
Adenosquamous carcinoma & 3 \\
Pathologic N stage & 36 \\
N1 & 69 \\
N2 & 6 \\
N3 & 111 \\
Total & \\
\hline
\end{tabular}

\section{Measuring the Size of Lymph Nodes}

In each lymph node station that was TP or FP with PET scan, the short-axis diameters of the largest lymph nodes were measured on CT scan.

\section{Measuring the Size of Metastasis}

The dissected lymph nodes were examined histologically by using formalin-fixed and paraffin-embedded sections with hematoxylin and eosin staining. Each lymph node was examined with 1 hematoxylin and eosin-stained section. In each lymph node station containing metastases, the lymph node with the largest metastatic lesions was selected, and the long-axis diameters of the metastatic foci in the lymph nodes were measured. The sizes of metastatic foci were measured with a microscope.

\section{Control Subjects}

Another 277 metastatic lymph nodes from 111 patients with lung cancer who had undergone operation with mediastinal lymph node dissection from 1985 to 2001 were also examined as a control in the same way: that is, (1) the lymph nodes with the largest metastatic lesions in each lymph node station were selected, and (2) the long-axis diameters of the metastatic foci were measured. The sizes were classified in 1-mm graduations from the smallerthan-1-mm class to the 10-mm-or-larger class. The histologic types were adenocarcinoma in 72 patients, squamous cell carcinoma in 26 , large cell carcinoma in 10, and adenosquamous carcinoma in 3 (Table 2). The pathologic classification was N1 in 36 patients, N2 in 69 , and N3 in 6.

\section{Statistical Analysis}

TP, true-negative (TN), FP, and FN results of PET and CT scanning for lymph node metastasis were compared with the results of pathologic diagnosis. Sensitivity was calculated as TP/TP + FN, specificity as TN/TN + FP, positive predictive value as TP/TP + $\mathrm{FP}$, negative predictive value as TN/TN $+\mathrm{FN}$, and accuracy as TP + TN/total. All data were analyzed for significance by using the 2-tailed Student $t$ test. All values in the text and tables are given as mean $\pm \mathrm{SD}$.

\section{Results}

The sensitivity and specificity in each CR value showed the optimal CR cutoff value to be 0.25 (Figure 1). Therefore,

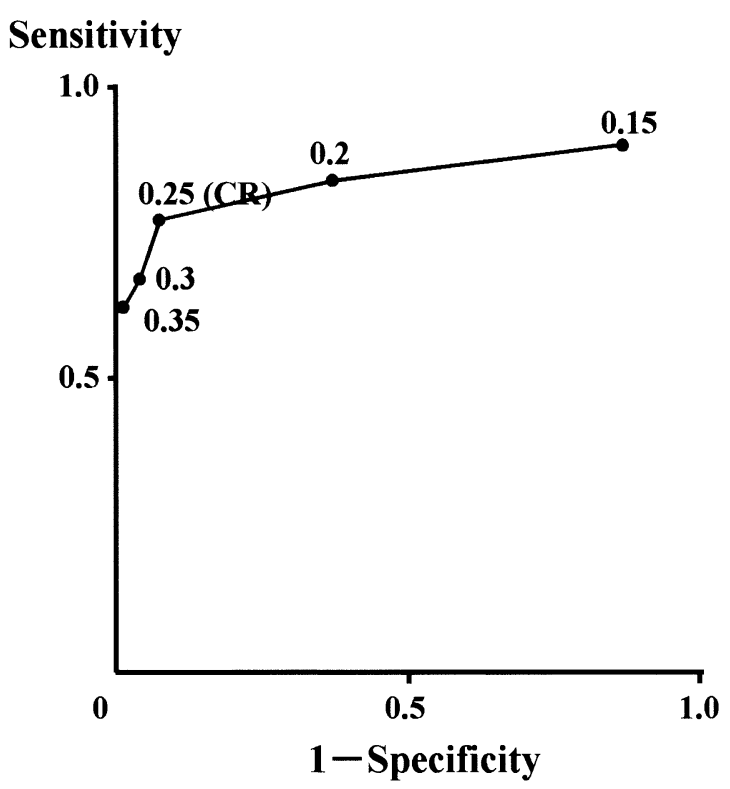

Figure 1. Receiver operating characteristic curve and contrast ratio (CR) value for diagnosing lymph node metastasis. The highest activities in the lymph node ROI (L) and in the cerebellum ROI (C) were measured. The CR was calculated by L/C in each lymph node station as an index of FDG uptake.

lymph nodes with a CR of 0.25 or higher were defined as positive for FDG and those with $\mathrm{CR}$ less than 0.25 as negative.

In the 564 lymph node stations examined, PET scanning yielded TP in 28 lymph node stations, FN in 8, FP in 10, and TN in 518 (Table 3). For the same lymph nodes, CT scanning yielded TP in 19 lymph node stations, FN in 17, FP in 8 , and TN in 520 (Table 4). PET and CT scanning showed a sensitivity of $78 \%$ and $53 \%$, a specificity of $98 \%$ and $98 \%$, an accuracy of $97 \%$ and $96 \%$, a positive predictive value of $74 \%$ and $70 \%$, and a negative predictive value of $98 \%$ and $97 \%$, respectively (Table 5). PET scanning showed significantly higher sensitivity than CT scanning $(P$ $=.026$ ), although there were no significant differences in specificity, accuracy, positive predictive value, or negative predictive value between the 2 diagnostic modalities. In the 8 FN lymph nodes with PET, 5 were located in the hilar lymph node station (\#12), 1 in the interlobar station (\#11), 1 in the tracheobronchial station (\#4), and 1 in the Botallo station (\#5).

Table 6 shows the correlation between $\mathrm{N}$ staging with PET and pathologic $\mathrm{N}$ staging. PET scanning correctly identified the $\mathrm{N}$ stage in 67 patients, whereas it showed understaging in 7 and overstaging in 6, of which the accuracy was $84 \%$. Table 7 shows the correlation between $\mathrm{N}$ staging with $\mathrm{CT}$ and pathologic $\mathrm{N}$ staging. CT scanning correctly identified the $\mathrm{N}$ stages in 60 patients, whereas it showed understaging in 14 patients and overstaging in 6 , of 
TABLE 3. Positron emission tomography analysis with pathologic diagnosis

\begin{tabular}{lllr}
\hline & \multicolumn{2}{c}{ No. of lymph node stations } \\
\cline { 2 - 4 } Variable & With metastasis & Without metastasis & Total \\
\hline PET diagnosis & & & \\
Positive & 28 true-positive & 10 false-positive & 38 \\
$\quad$ Negative & 8 false-negative & 518 true-negative & 526 \\
Total & 36 & 528 & 564 \\
\hline
\end{tabular}

PET, Positron emission tomography.

TABLE 4. Computed tomography analysis with pathologic diagnosis

\begin{tabular}{llrr}
\hline & \multicolumn{2}{c}{ No. of lymph node stations } \\
\cline { 2 - 4 } Variable & With metastasis & Without metastasis & Total \\
\hline CT diagnosis & & \\
$\quad$ Positive & 19 true-positive & 8 false-positive & 27 \\
$\quad$ Negative & 17 false-negative & 520 true-negative & 537 \\
Total & 36 & 528 & 564 \\
\hline
\end{tabular}

$C T$, Computed tomography.

TABLE 5. Diagnostic results of PET and CT scanning

\begin{tabular}{lccc}
\hline Variable & PET & CT & Difference \\
\hline Sensitivity & 0.78 & 0.53 & $P=.026$ \\
Specificity & 0.98 & 0.98 & 0.63 \\
Accuracy & 0.97 & 0.96 & 0.28 \\
Positive predictive value & 0.74 & 0.70 & 0.77 \\
Negative predictive value & 0.98 & 0.97 & 0.08 \\
\hline
\end{tabular}

PET, Positron emission tomography; $C T$, computed tomography.

TABLE 6. Correlation of $\mathbf{N}$ staging with positron emission tomography and pathologic $\mathrm{N}$ stage

\begin{tabular}{crrrrr}
\hline & \multicolumn{4}{c}{ Pathologic N stage } & \\
\cline { 2 - 5 } PET diagnosis & N0 & N1 & N2 & N3 & Total \\
\hline N0 & 52 & 5 & 2 & 0 & 59 \\
N1 & 3 & 4 & 0 & 0 & 7 \\
N2 & 1 & 1 & 8 & 0 & 10 \\
N3 & 0 & 0 & 1 & 3 & 4 \\
Total & 56 & 10 & 11 & 3 & 80
\end{tabular}

PET, Positron emission tomography.

which accuracy was $75 \%$. There was no significant difference of accuracy in overall $\mathrm{N}$ staging between the PET and CT scanning $(P=.17)$. However, PET scanning could identify $8(73 \%)$ of 11 with N2 disease, which was significantly more accurate than $3(27 \%)$ of 11 with CT $(P=$ .033). There was no significant difference of diagnosis for N1 disease between the 2 .

The sizes of the metastatic foci in the lymph nodes that yielded FN results with PET scanning ranged from 0.5 to 9
TABLE 7. Correlation of $\mathbf{N}$ staging with computed tomography and pathologic $\mathbf{N}$ stage

\begin{tabular}{crrrrr}
\hline & \multicolumn{4}{c}{ Pathologic N stage } \\
\cline { 2 - 5 } CT diagnosis & N0 & N1 & N2 & N3 & Total \\
\hline N0 & 52 & 7 & 6 & 0 & 65 \\
N1 & 2 & 2 & 1 & 0 & 5 \\
N2 & 2 & 1 & 3 & 0 & 6 \\
N3 & 0 & 0 & 1 & 3 & 4 \\
Total & 56 & 10 & 11 & 3 & 80 \\
\hline
\end{tabular}

$C T$, Computed tomography.

$\mathrm{mm}$, with a mean value of $3 \pm 1 \mathrm{~mm}$, whereas those that yielded TP results ranged from 4 to $18 \mathrm{~mm}$, with a mean value of $10 \pm 3 \mathrm{~mm}$ (Figure 2). There was a significant difference in the size of metastatic foci between the FN and TP lymph nodes $(P<.001)$. PET scanning could detect none of the metastatic foci smaller than $4 \mathrm{~mm}$, but it could detect all of the metastatic foci $5 \mathrm{~mm}$ or larger, except for 1 FN lymph node that had a metastatic lesion of carcinosarcoma $9 \mathrm{~mm}$ in size. Metastasis in a single node was found in $15(54 \%)$ of the $28 \mathrm{TP}$ lymph node stations and in 5 (50\%) of 10 FN lymph node stations; the difference between the 2 was nonsignificant. None of the FN lymph nodes had metastasis of bronchioloalveolar carcinoma, which usually showed FN with PET scanning. The sizes of the lymph nodes that yielded FP results on PET scanning ranged from 9 to $16 \mathrm{~mm}$, with a mean value of $12 \pm 2 \mathrm{~mm}$, whereas those that yielded TP results ranged from 6 to 15 $\mathrm{mm}$, with a mean value of $10 \pm 2 \mathrm{~mm}$ (Figure 3). The former was significantly larger than the latter $(P<.01)$. None of the FP lymph nodes was smaller than $9 \mathrm{~mm}$. All of the FP lymph nodes had histologic findings of reactive lymph adenitis.

Table 8 shows the distribution in sizes of metastatic foci in the 277 metastatic lymph nodes of the 111 patients in Table 2. Eighty-nine lymph nodes (32\%) had metastatic foci smaller than $4 \mathrm{~mm}$. Of the 111 patients, $34(31 \%)$ had metastatic foci smaller than $4 \mathrm{~mm}$.

\section{Discussion}

Whereas a standard uptake value (SUV) is used for diagnosis of malignancy, we evaluated the activity ratio with CR instead of SUV for the following reasons: (1) SUV is dependent on the dose of FDG and the time of measurement after injection of FDG, and (2) hyperglycemia in diabetic patients decreases both the blood clearance of FDG and the accumulation of FDG in tumor tissue. Actually, the mean SUV of malignant pulmonary nodules has been report$\mathrm{ed}^{10-14}$ to range from 5.5 to 10.1 . Several criteria have been used to detect lymph node metastases of lung cancer by using PET scanning, including accumulation of FDG without objective criteria, ${ }^{6,15}$ accumulation greater than medias- 
tinal blood flow, ${ }^{2-4}$ and $\mathrm{CR}$ with the paravertebral muscles. ${ }^{16}$ We evaluated the accumulation of FDG by using the activity ratio in comparison to the cerebellum, because the accumulation of FDG in the cerebellum is more stable than that in mediastinal blood flow or in muscle. We found that the cutoff value of 0.25 or larger was the most reliable for diagnosing lymph node metastasis with PET scanning.

This study showed that the PET scan could not detect lymph nodes that had metastatic foci smaller than $4 \mathrm{~mm}$. Because there was no significant difference of frequency of single-node metastasis between the TP and FN lymph node stations, the sensitivity of PET for $\mathrm{N}$ staging could be dependent on the size of metastatic foci rather than on the number of metastatic lymph nodes in each station. The FDG uptake of small lesions may have been underestimated as a result of the spatial resolution of the PET scanner, eg, because of the partial-volume effect. Although Gupta and colleagues $^{3}$ reported that the sensitivity, specificity, and accuracy of PET scanning for detecting lymph node metastasis showed no difference between lymph nodes smaller than $1 \mathrm{~cm}, 1$ to $3 \mathrm{~cm}$, or larger than $3 \mathrm{~cm}$, they did not define the lower size limit for PET scanning. To examine the general distribution of general sizes of metastatic foci within the lymph nodes, we measured the sizes of 277 metastatic lymph nodes in a large series of lung cancer patients. If $4 \mathrm{~mm}$ were the lower limit for PET scanning to detect metastatic foci, 89 (32\%) of lymph nodes with metastases smaller than $4 \mathrm{~mm}$ would yield FN results with PET scanning. Even if $3 \mathrm{~mm}$ were the lower limit, 59 (21\%) of lymph nodes with metastases smaller than $3 \mathrm{~mm}$ would yield FN results (Table 8). Considering the spatial resolution of the PET scanner, artifacts resulting from respiratory movement, image reconstruction, and biological effects, it is difficult to evaluate such small metastatic foci. Current PET scanners, similar to the POSICAM used in this study, achieve transaxial resolutions of 5 to $6 \mathrm{~mm}$ at full width, half maximum. Tumors smaller than $10 \mathrm{~mm}$ could therefore not be resolved even by the current scanner because of the partial-volume effect. It has in fact been reported that the current generation of PET scanners are unable to delineate lung cancers smaller than $10 \mathrm{~mm} .{ }^{17}$ This study did show, however, that all metastatic foci $5 \mathrm{~mm}$ or larger, except 1 with the lesion $9 \mathrm{~mm}$ in size, were detected with PET scanning, apparently exceeding the lower limit of spatial resolution. We consider that metastatic foci smaller than 10 $\mathrm{mm}$ can be detected with PET scanning for the following reasons: (1) metastatic tumor cells would have more biological activity than primary tumor cells, causing higher FDG uptake in metastatic foci than in the primary sites, and (2) the immune reaction of macrophages within metastatic lymph nodes might increase FDG uptake, because FDG has been reported to accumulate not only in tumor cells, but also in macrophages. ${ }^{18}$

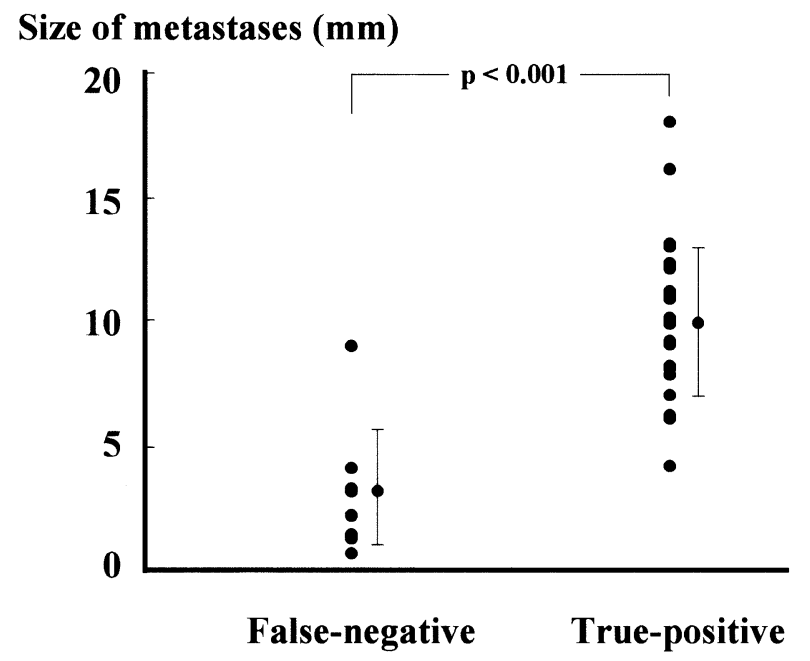

Figure 2. The distribution of sizes of metastatic foci in falsenegative and true-positive lymph nodes with PET scan.

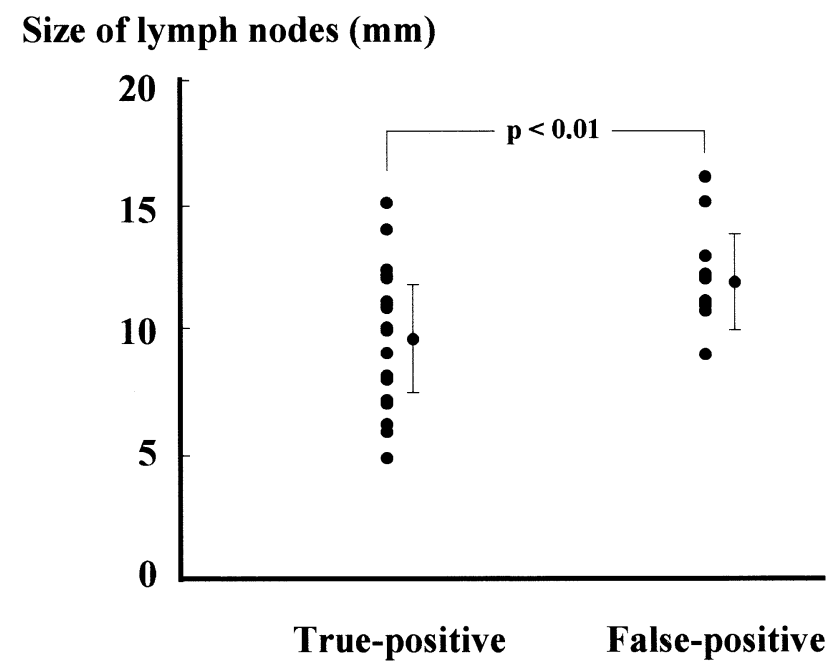

Figure 3. The distribution of sizes of metastatic true-positive and false-positive lymph nodes with PET scan.

TABLE 8. Distribution of sizes of metastatic foci in the 277 metastatic lymph nodes

\begin{tabular}{|c|c|c|c|c|c|c|c|c|c|c|c|}
\hline \multirow[b]{2}{*}{ Variable } & \multicolumn{11}{|c|}{ Size $(\mathrm{mm})$} \\
\hline & $<1<2$ & $2<3$ & $<4$ & $<5$ & $<6$ & $<7$ & $<8$ & $<9$ & $<10$ & $\geq 10$ & Total \\
\hline No. & 1432 & 59 & 89 & 110 & 125 & 138 & 151 & 167 & 190 & 87 & 277 \\
\hline$\%$ & $\begin{array}{ll}5 & 12\end{array}$ & 21 & 32 & 40 & 45 & 50 & 55 & 60 & 69 & 31 & 100 \\
\hline
\end{tabular}

In this study, we excluded central-type lung cancer patients for the following reasons: (1) it is difficult for PET scanning to distinguish the primary tumor from adjacent nodal metastasis, and (2) bronchial obstruction by a central tumor can lead to inflammation in the locoregional lymph 
nodes, with subsequent FDG uptake. Nevertheless, 6 (75\%) of 8 FN lymph node stations with PET scanning were located in the hilar region. Although there was no significant difference of accuracy for overall $\mathrm{N}$ staging between PET and CT scanning, PET could identify N2 disease more accurately than CT. Vesselle and associates ${ }^{19}$ also reported that, although PET scanning could correctly differentiate $\mathrm{N} 0$ or N1 from $\mathrm{N} 2$ or N3 disease, it could not reliably identify N1 disease, with only 6 of 21 cases identified. We therefore believe that PET scanning is useful for determining an indication of operation or neoadjuvant therapy for N2 disease but that it is not reliable for N1 disease.

It has been reported that FP results for lymph nodes can occur with PET scanning because of concurrent inflammatory conditions or centrally located lung cancer. ${ }^{20}$ Although this study excluded centrally located lung cancers, it is well known that lymph nodes near the primary tumor could become enlarged with an immune reaction, which could become positive with PET scanning because of FDG uptake by reactive macrophages, resulting in an FP result. This study showed that the FP lymph nodes with PET scanning were significantly larger than the TP ones and that none of the FP lymph nodes was smaller than $9 \mathrm{~mm}$. Therefore, we consider that positive lymph nodes with PET scanning smaller than $9 \mathrm{~mm}$ are more likely to be TP than FP.

Although PET scanning is superior to CT scanning for $\mathrm{N}$ staging in lung cancer, it was unable to detect metastatic foci smaller than $4 \mathrm{~mm}$, which were not unusual sizes for metastatic lymph nodes in lung cancer. Positive lymph nodes with PET scanning that are smaller than $9 \mathrm{~mm}$ are more likely to be TP than FP.

\section{References}

1. Dwamena BA, Sonnad SS, Angobaldo JO, Wahl RL. Metastases from non-small cell lung cancer: mediastinal staging in the 1990s-metaanalytic comparison of PET and CT. Radiology. 1999;213:530-6.

2. Gupta NC, Tamin WJ, Graeber GG, Bishop HA, Hobbs GR. Mediastinal lymph node sampling following positron emission tomography with fluorodeoxyglucose imaging in lung cancer staging. Chest. 2001; 120:521-7.

3. Gupta NC, Geoffrey M, Graeber GG, Bishop HA. Comparative efficacy of positron emission tomography with fluorodeoxyglucose in evaluation of small $(<1 \mathrm{~cm})$, intermediate $(1$ to $3 \mathrm{~cm})$, and large $(>3$ cm) lymph node lesions. Chest. 2000;117:773-8.
4. Graeter TP, Hellwig D, Hoffman K, Ukena D, Kirsch CM, Schafers HJ. Mediastinal lymph node staging in suspected lung cancer: comparison of positron emission tomography with F-18-fluorodeoxyglucose and mediastinoscopy. Ann Thorac Surg. 2003;75:231-6.

5. Chin R Jr, Ward R, Keyes JW, et al. Mediastinal staging of non-small cell lung cancer with positron emission tomography. Am J Respir Crit Care Med. 1995;152:2090-6.

6. Bury T, Paulus P, Dowlati A, Corhay JL, Weber T, Ghaye B, et al. Staging of the mediastinum: value of positron emission tomography imaging in non-small cell lung cancer. Eur Respir J. 1996;9:2560-4.

7. Valk PE, Pounds TR, Hopkins DM, Haseman MK, Hofer GA, Greiss $\mathrm{HB}$, et al. Staging non-small cell lung cancer by whole-body positron emission tomography imaging. Ann Thorac Surg. 1995;60:1573-82.

8. Naruke T, Suemasu K, Ishikawa S. Lymph node mapping and curability at various levels of metastasis in resected lung cancer. $J$ Thorac Cardiovasc Surg. 1978;76:832-9.

9. Moses LE, Shapiro D, Littenberg B. Combining independent studies of a diagnostic test into a summary ROC curve: data-analytic approaches and some additional considerations. Stat Med. 1993;12:1293-316.

10. Scott WJ, Schwabe JL, Gupta NC, et al. Positron emission tomography of lung tumors and mediastinal lymph nodes using $\left[{ }^{18} \mathrm{~F}\right]$ fluorodeoxyglucose. Ann Thorac Surg. 1994;58:698-703.

11. Gupta NC, Maloof J, Gunel E. Probability of malignancy in solitary pulmonary nodules using fluorine-18-FDG and PET. J Nucl Med. 1996;37:943-8.

12. Dewan NA, Gupta NC, Redepenning LS, et al. Diagnostic efficacy of PET-FDG imaging in solitary pulmonary nodules. Potential role in evaluation and management. Chest. 1993;104:997-1002.

13. Imdahl A, Jenkner S, Brink I, et al. Validation of FDG positron emission tomography for differentiation of unknown pulmonary lesions. Eur J Cardiothorac Surg. 2001;20:324-9.

14. Lowe VJ, Fletcher JW, Gobar L, et al. Prospective investigation of positron emission tomography in lung nodules. J Clin Oncol. 1998; 16:1075-84.

15. Poncelet AJ, Lonneux M, Coche E, Weynand B, Noirhomme P. PET-FDG scan enhances but does not replace preoperative surgical staging in non-small cell lung carcinoma. Eur J Cardiothorac Surg. 2001;20:468-75.

16. Yasukawa T, Yoshikawa K, Aoyagi H, Yamamoto N, Tamura K, Suzuki K, et al. Usefulness of PET with ${ }^{11} \mathrm{C}$-methionine for the detection of hilar and mediastinal lymph node metastasis in lung cancer. J Nucl Med. 2000;41:283-90.

17. Tan BB, Flaherty KR, Kazerooni EA, Iannettoni MD. The solitary pulmonary nodule. Chest. 2003;123:89S-96S.

18. Kubota R, Yamada S, Kubota K, Ishikawa K, Tamahashi N, Ito T. Intratumoral distribution of fluorine-18-fluorodeoxyglucose in vivo: high accumulation in macrophages and granulation tissues studied by microautoradiography. J Nucl Med. 1992;33:1972-80.

19. Vesselle H, Pugsley JM, Vallieres E, Wood DE. The impact of fluorodeoxyglucose F 18 positron-emission tomography on the surgical staging of non-small cell lung cancer. J Thorac Cardiovasc Surg. 2002;124:511-9.

20. Roberts PF, Follette DM, Haag D, Park JA, Valk PE, Pounds TR, et al. Factors associated with false-positive staging of lung cancer by positron emission tomography. Ann Thorac Surg. 2000;70:1154-60. 